\title{
Social determinants and behaviors associated with overweight and obesity among youth and adults in a peri-urban area of Maputo City, Mozambique
}

Ivalda Macicame ${ }^{1,2}$, António

Prista $^{3}$, Klaus G Parhofer ${ }^{4}$, Nílzio

Cavele $^{1}$, Cremildo Manhiça ${ }^{1}$,

Sheila Nhachungue ${ }^{1}$, Elmar

Saathoff5,6, Eva Rehfuess ${ }^{7}$

${ }^{1}$ Instituto Nacional de Saúde, Maputo

City, Mozambique

${ }^{2}$ Center for International Health $(\mathrm{CIH})$,

Ludwig-Maximilians-Universität München (LMU) Munich, Germany

${ }^{3}$ Universidade Pedagógica, Maputo City, Mozambique

${ }^{4}$ Medizinische Klinik IV - Grosshadern, Klinikum der Ludwig-MaximiliansUniversität München (LMU), Munich, Germany

${ }^{5}$ Division of Infectious Diseases and Tropical Medicine, University Hospital, LMU, Munich, Germany

${ }^{6}$ German Centre for Infection Research (DZIF), partner site Munich, Munich, Germany

${ }^{7}$ Institute of Medical Information Processing, Biometry and Epidemiology, Pettenkofer School of Public Health, LMU Munich, Munich, Germany
Background Overweight and obesity are important risk factors for non-communicable diseases (NCDs) such as cardiovascular diseases (CVD), type 2 diabetes and certain cancers. NCDs are responsible for an increased number of deaths worldwide, including in developing countries. We aimed to determine the prevalence of overweight and obesity among youth and adults in a peri-urban area of Maputo city, Mozambique, and to assess their social and behavioral determinants.

Methods A cross-sectional study was conducted in a Health and Demographic Surveillance System (HDSS) area in Maputo city. We measured BMI and interviewed 15-64-year-old inhabitants to assess sociodemographic and behavioral characteristics using the STEPwise Approach methodology. A household wealth index was derived through Principal Component Analysis of various household assets and physical activity (PA) was measured using pedometers and accelerometers. Univariable and multivariable analyses were conducted to determine associations between overweight/obesity and social and behavioral determinants.

Results Among a total of 931 participants, the prevalence of overweight $\left(\mathrm{BMI} \geq 25 \mathrm{~kg} / \mathrm{m}^{2}\right)$ and obesity $\left(\mathrm{BMI} \geq 30 \mathrm{~kg} / \mathrm{m}^{2}\right)$ was $30.9 \%$ (95\% confidence interval $(\mathrm{CI})=28.0,33.9)$ and $12.6 \%(95 \% \mathrm{CI}=10.4,14.7)$, respectively; one in every 10 youths and adults were underweight. Being female, older and living in a wealthier household were found to be significantly associated with overweight and obesity. Those with higher levels of education were found to have a reduced risk of being obese compared to those with no or lower levels of education. Behavioral risk factors (diet, alcohol and tobacco consumption and physical activity) did not significantly increase the risk of overweight and obesity.

Conclusions Overweight and obesity are highly prevalent in this peri-urban part of the Mozambican capital, where underweight is still present in youth and adults, confirming that the country is facing a double burden of malnutrition. Social determinants of health should be taken into consideration in the design and implementation of NCD prevention programs.

\section{Correspondence to:}

Ivalda Macicame, MD

Armando Tivane Avenue, \# 373, 15th floor

Maputo City

Mozambique

Ivalda.macicame@gmail.com
Globally, non-communicable diseases (NCDs), notably cardiovascular disease (CVD), diabetes, chronic respiratory disease and cancers, currently cause more deaths than all other causes combined [1]. The prevalence of overweight and obesity, which are important risk factors for NCDs such as CVD, type 2 diabetes and 
certain cancers, has more than doubled during the last three decades worldwide [1]. Although one of the objectives of the Global Action Plan for the prevention and control of NCDs is to tackle the modifiable risk factors for NCDs and their underlying social determinants through the creation of health-promoting environments, research on the social determinants of health is rarely done in low-income countries [2]. Monitoring the trends of overweight and obesity and understanding their determinants, particularly in urban settings from low-income countries, is crucial for achieving this objective [3].

In 2016 adults in low- and middle-income countries had almost twice the risk of dying from NCDs compared to those from high-income countries [4] and the prevalence of obesity in the African region was estimated to be about $15 \%$ in women and $6 \%$ in men [1].

According to the WHO, NCDs are currently responsible for approximately 32\% of deaths in Mozambique [5]. Over the past few decades, the prevalence of overweight and obesity among Mozambican youth [6] and adults [7] has increased. According to the last STEPS survey, undertaken in 2014/2015, to assess risk factors for NCDs conducted in Mozambique, the prevalence of overweight increased from 18.3\% to 30.5\% in women and from $11.7 \%$ to $18.2 \%$ in men compared to the STEPS survey undertaken in 2005. Similarly, over the same time period the prevalence of obesity increased from $7 \%$ to $13 \%$ in women and from $2 \%$ to $5 \%$ in men. A higher prevalence was observed in urban than in rural areas $[7,8]$.

Rapid population growth in urban settings, mostly through in-migration from rural areas to peri-urban areas, is leading people from low-income countries such as Mozambique to live in precarious conditions [6]. This rampant and unplanned urbanization, characterized by a lack of open space and an increase in criminality as distinctive features of African urban centers, is coinciding with decreasing physical activity levels [9] and increasing obesity and its comorbidities [10]. The objective of this study was to determine the prevalence of overweight and obesity among youth and adults in a peri-urban area of Maputo city, Mozambique, and to assess their social and behavioral determinants.

\section{METHODS}

\section{Study population and sampling procedure}

Mozambique is a low-income country located on the east coast of southern Africa. A Health and Demographic Surveillance System (HDSS) is being implemented in a peri-urban area of Maputo city, Polana Caniço, with about 92000 inhabitants living in an area of $6.8 \mathrm{~km}^{2}$ [11]. The HDSS has enrolled approximately $15000 \mathrm{in}-$ habitants (from 20 randomly selected blocks) between January and June 2017. Figure 1 shows the HDSS Polana Caniço surveillance area.

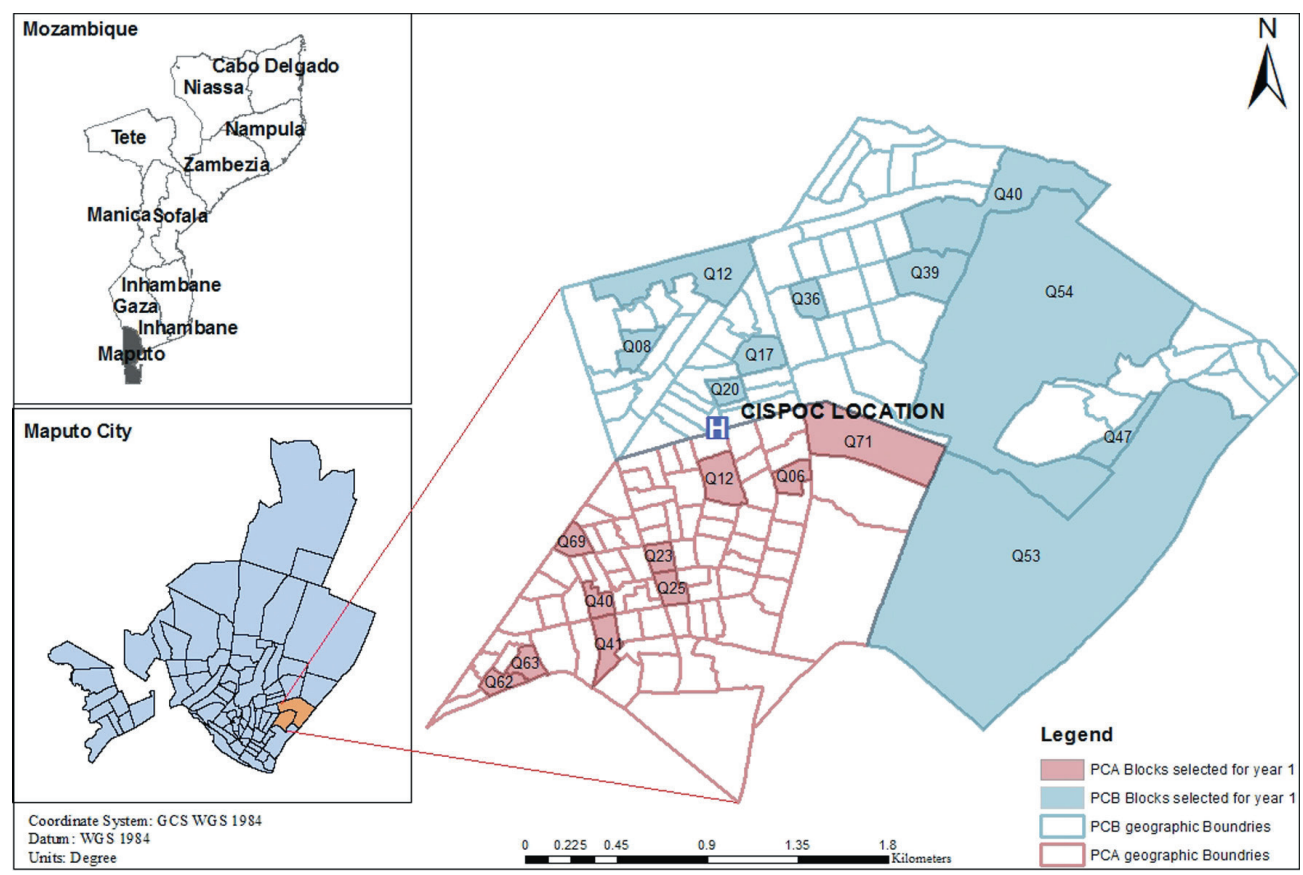

Figure 1. HDSS Polana Caniço surveillance area. 
From October 2017 to April 2018 we recruited 15-64 year-old males and non-pregnant females from randomly selected households from the HDSS. Given the unknown prevalence of risk factors for NCDs specifically for Maputo city, a prevalence of 50\% was used to calculate the required sample size in a population of 92000 inhabitants. Due to possible physiological / metabolic differences between men and women, the sample size was doubled in order to allow for gender-disaggregated analyses. Additionally, the sample was increased by $10 \%$ in order to compensate for the nature of the study (data collection at the community level) where blank responses and loss of quality of biological samples were likely to occur. This resulted in a sample size of 843 individuals, corresponding to a minimum of 282 households. To overcome issues related to household unavailability or refusals, an additional 282 households were randomly selected as replacements. Community leaders (secretariats of neighbourhoods, heads of blocks and other trusted individuals from the community) supported the study team with the community sensitization through six community meetings and door-to-door awareness-raising activities. Community leaders also scheduled the visit to the selected households. In each selected household, all individuals aged 15 to 64 years were eligible for enrolment into the study. After three attempted visits, if eligible individuals were not available, surrogate households were approached.

\section{Data collection}

Two questionnaires - a household questionnaire and a demographic and risk factor questionnaire - were administered by trained interviewers in Portuguese or in a local language (XiChangana or Ronga); physical measurements were taken by the same trained interviewers.

The household questionnaire, based on Vyas and Kumaranayake [12], was completed by the head of household or his/her representative and included questions regarding household assets, type of hygiene facilities, construction materials and other relevant information to determine a setting-specific household wealth index. The demographic and risk factor questionnaire, based on the STEPwise Approach [13] and modified to fit the specific study objectives, was completed by each study participant. This covered questions related to age, gender, place of birth, education level, mother tongue, knowledge of Portuguese and occupation; as well as questions related to diet, physical activity and tobacco and alcohol consumption. Participant height was measured in centimeters using a stadiometer (SECA 2013, SECA Group, Hamburg, Germany) and weight in kilograms using a balance (OMRON HN289, OMRON HealthCare, Yangzhou, China). Participants with a Body Mass Index (BMI) of $30 \mathrm{~kg} / \mathrm{m}^{2}$ or above were referred for nutritional counseling at a health facility, with all health facilities in the area offering this service. To measure physical activity, the number of steps and the time spent in moderate to vigorous physical activity (MVPA) were recorded using pedometers (STEPmvx, Guangdong, China) for seven consecutive days, except when sleeping and while taking a bath. No financial or material compensation was provided to participants.

This study was conducted according to the ethical principles described in the Helsinki Declaration and its revisions [14]. It was approved by the Instituto Nacional de Saúde (National Institute of Health - INS) Institutional Review Board and the National Health Bioethics Committee of Mozambique, as well as the Ethics Board of the Faculty of Medicine of Ludwig-Maximilians-Universität München, Germany. The voluntary participation of each adult household member was documented by written informed consent. For the voluntary participation of individuals between the ages of 15 and 17, a written assent form was administered to the minor and written informed consent was provided by the minor's legal representative. The findings of this study are being reported in accordance with the STROBE statement [15].

\section{Statistical analysis}

A conceptual framework (Figure 2) was developed to describe the relationships between four NCDs (cardiovascular diseases, diabetes, cancer and chronic respiratory disease) and their underlying risk factors, including non-modifiable, metabolic and behavioral risk factors as well as their broader environmental and social determinants. With respect to behavioral risk factors, we focused on those for which the link with overweight and obesity can be considered causal, ie, an unhealthy diet, insufficient physical activity, alcohol consumption and tobacco use [16]. The analysis was mostly determined a priori, drawing on the conceptual framework and focusing on non-modifiable determinants, social determinants and behavioral risk factor as predictors of overweight and obesity.

Questionnaires were designed using the Open Data Kit (ODK) Collect 1.14.0 app (OpenRosa Consortium, University of Washington, Seattle, USA) and data were entered electronically using tablet computers (Samsung Galaxy TAB E 9.6 inches, Seoul, South Korea). Data were exported to an ODK aggregate 1.4.13 server using a mysql 5.7.1 database. Data checking and cleaning was conducted independently by a staff trained on 


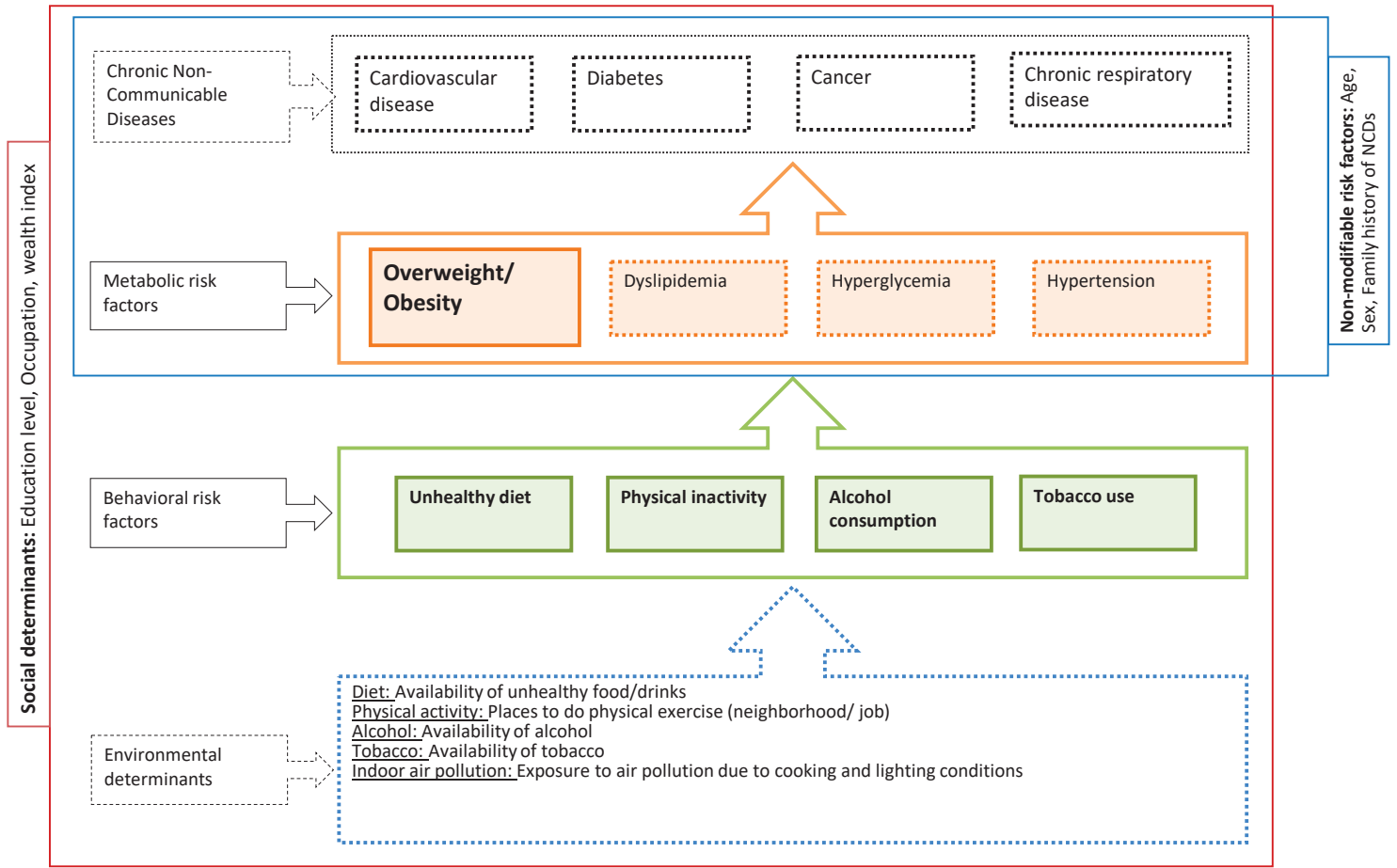

Figure 2. Conceptual framework of risk factors for overweight and obesity and related health outcomes.

quality assurance and control and by a statistician using Excel 2013 and R Studio version 3.4.2 (R Foundation, Boston, MA, USA). Data were subsequently exported to Stata version 15.1 (StataCorp LLC, College Station, Texas, USA) for analysis.

Socio-demographic characteristics of study participants are described using medians and inter quartile ranges (IQR) for continuous variables and proportions for categorical variables, for all participants and stratified by gender. A household wealth index was generated using a modification of an approach initially described by Filmer and Pritchett $[17,18]$. This method uses principal component analysis to combine data regarding ownership of various household assets, building materials of the house and other indicators of household wealth into a single index, which is then issued as a categorical variable (quintiles). Body Mass Index (BMI) calculated as weight (in $\mathrm{kg}$ ) over (height (in $\left.\mathrm{m}^{2}\right)$, was categorized as underweight $\left(<18.5 \mathrm{~kg} / \mathrm{m}^{2}\right)$, normal weight $(18.5$ to $\left.<25 \mathrm{~kg} / \mathrm{m}^{2}\right)$, overweight $\left(\geq 25\right.$ to $\left.<30 \mathrm{~kg} / \mathrm{m}^{2}\right)$ and obese $\left(\geq 30 \mathrm{~kg} / \mathrm{m}^{2}\right)$ according to the World Health Organization (WHO) [19]. The level of physical activity was determined by the number of steps and the time spent in moderate to vigorous physical activity (MVPA) per day using cutoff values of 10000 steps per day and 30 minutes per day, respectively. $\chi^{2}$-testing was used to examine differences in BMI distribution and behavioral risk factors for NCDs between males and females.

Associations of various potential risk factors with the binary outcome overweight (BMI $\geq 25$, which includes obese patients and is called "overweight+" below) were analyzed using log-link binomial regression and Poisson regression $[20,21]$ with robust variance estimates to adjust for within household clustering. In a first step, univariable regressions adjusted for age and gender were used to identify factors whose association with overweight + had a $P$-value $(P) \leq 0.1$ to be considered for inclusion into multivariable models. Based on the univariable results, three different multivariable models were developed according to the type of risk factor (socioeconomic factors, behavioral risk factors, behavioral and socioeconomic factors combined) shown in Figure 2. Decisions on which variables to retain in these multivariable models were guided by the Akaike Information Criterion (AIC) and the most parsimonious model was chosen. If two or more variables that qualified for inclusion were too collinear to be included in the same model (variance inflation factor $\geq 10$ ) [22], the variable that resulted in the lowest AIC was used. A final model was constructed to combine the above identified factors into one model. Again the most parsimonious model according to the AIC was chosen. Gender, age and household wealth index were included in all multivariable models to adjust for potential confounding.

The procedures described above were also used to analyze the associations of various potential risk factors with the binary outcome obesity ( $\mathrm{BMI} \geq 30$ ), using a more relaxed $P$-value of $\leq 0.2$ for inclusion into multivariable models. 


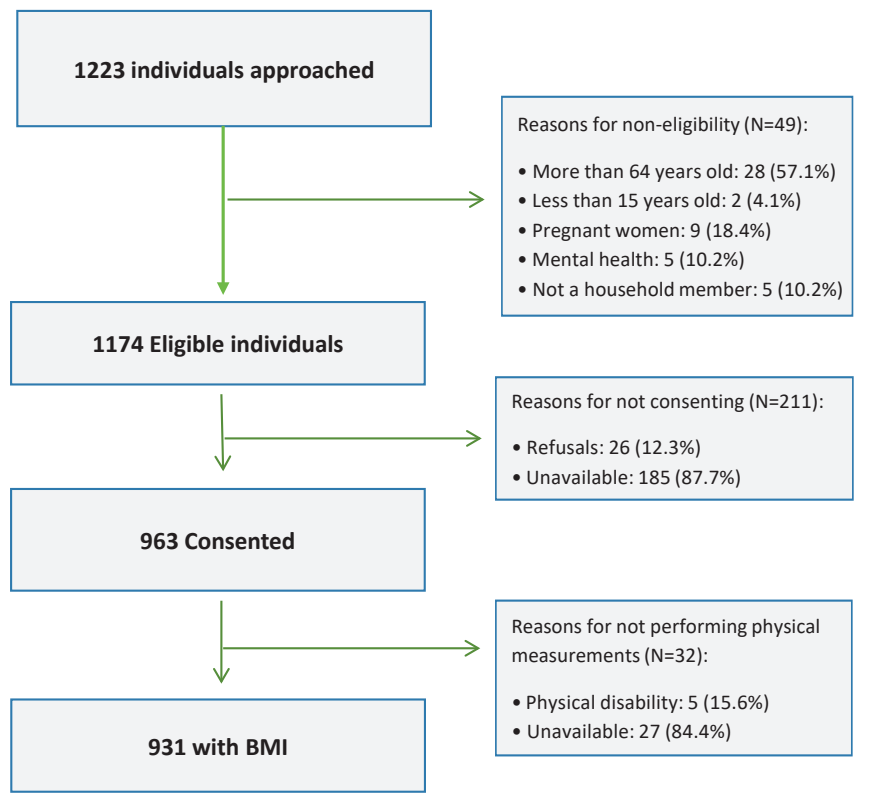

Figure 3. Screening and enrolment of participants.

\section{RESULTS}

Overall 1223 individuals living in 367 households (mean of three persons per household, with a minimum of one and a maximum of 13 adults per household) were approached, of whom 1174 individuals (96\%) were eligible for inclusion in the study. Among the eligible participants, 963 (82\%) at least one member of the 367 households -consented to participate. From the consented participants 32 (3.3\%) were excluded from this analysis for not having BMI data. Reasons for non-eligibility and for not consenting are presented in Figure 3. Socio-demographic characteristics of study participants are presented in Table 1. Among 931 participants with BMI data, 394 (42.3\%) were males and 537 (57.7\%) were females. The median age was $28(\mathrm{IQR}=21,41)$ years and half of the study participants (51\%) were single. More than half of the participants (53.6\% total, $61.9 \%$ for males and $47.5 \%$ for females) had at least completed primary education and $54.2 \%$ (69.9\% for males and $42.3 \%$ for females) were employed. Portuguese, the official language in Mozambique, was considered as their mother tongue by $28.4 \%$ of the participants and $88 \%$ reported that they can speak, write and read Portuguese. A good command of Portuguese may play a role, as the dissemination of official health information in Mozambique tends to take place in Portuguese, the country's official language.

Table 1. Socio-demographic characteristics of study participants $(\mathrm{N}=931)$

\begin{tabular}{|c|c|c|c|c|c|c|}
\hline \multirow{2}{*}{ CHARACTERISTICS } & \multicolumn{2}{|c|}{ TOTAL ( $\mathrm{N}=931)$} & \multicolumn{2}{|c|}{ MALE (N = 394) } & \multicolumn{2}{|c|}{ FEMALE (N = 537) } \\
\hline & $\mathrm{N}$ & $\%$ & $\mathbf{n}$ & $\%$ & $\mathbf{n}$ & $\%$ \\
\hline Age, years; median (IQR) & \multicolumn{2}{|c|}{$28(21,41)$} & \multicolumn{2}{|c|}{$27(20,38)$} & \multicolumn{2}{|c|}{$29(21,43)$} \\
\hline Age, years: & 922 & & 391 & & 531 & \\
\hline $15-24$ & 372 & $(40.4)$ & 165 & $(42.2)$ & 207 & $(39.0)$ \\
\hline $25-34$ & 223 & $(24.2)$ & 104 & $(26.6)$ & 119 & $(22.4)$ \\
\hline $35-44$ & 133 & $(14.4)$ & 57 & $(14.6)$ & 76 & $(14.3)$ \\
\hline $45-54$ & 121 & $(13.1)$ & 39 & $(10.0)$ & 82 & $(15.4)$ \\
\hline $55-64$ & 73 & $(7.9)$ & 26 & $(6.7)$ & 47 & $(8.5)$ \\
\hline Religion: & 869 & & 364 & & 505 & \\
\hline Muslim & 30 & $(3.5)$ & 16 & $(4.2)$ & 13 & $(2.5)$ \\
\hline Zion & 132 & $(15.2)$ & 42 & $(11.1)$ & 82 & $(15.6)$ \\
\hline Christian Catholic & 168 & $(19.3)$ & 78 & $(20.7)$ & 86 & $(16.3)$ \\
\hline Christian Protestant & 474 & $(54.6)$ & 175 & $(46.4)$ & 293 & $(55.6)$ \\
\hline No religion & 65 & $(7.5)$ & 39 & $(10.3)$ & 25 & $(4.7)$ \\
\hline Marital status: & 923 & & 391 & & 532 & \\
\hline Single & 471 & $(51.0)$ & 216 & $(55.2)$ & 255 & $(47.9)$ \\
\hline Marriage/Consensual union & 393 & $(42.6)$ & 165 & $(42.2)$ & 228 & $(42.9)$ \\
\hline Divorced/widowed & 59 & $(6.4)$ & 10 & $(2.6)$ & 49 & $(9.2)$ \\
\hline Mother tongue: & 922 & & 390 & & 532 & \\
\hline Portuguese & 262 & $(28.4)$ & 116 & $(29.7)$ & 146 & $(27.4)$ \\
\hline Bitonga & 25 & $(2.7)$ & 11 & $(2.8)$ & 14 & $(2.6)$ \\
\hline Xichangana & 458 & $(49.7)$ & 169 & $(43.3)$ & 289 & $(54.3)$ \\
\hline Xisena & 4 & $(0.4)$ & 1 & $(0.3)$ & 3 & $(0.6)$ \\
\hline Xirhonga & 20 & $(2.2)$ & 7 & $(1.8)$ & 13 & $(2.4)$ \\
\hline Xichope & 6 & $(0.7)$ & 1 & $(0.3)$ & 5 & $(0.9)$ \\
\hline Xitshwa & 35 & (3.8) & 19 & $(4.9)$ & 16 & $(3.0)$ \\
\hline Ndau & 22 & (2.4) & 16 & $(4.1)$ & 6 & $(1.1)$ \\
\hline Other & 90 & $(9.8)$ & 50 & $(12.8)$ & 40 & $(7.5)$ \\
\hline Education: & 922 & & 391 & & 531 & \\
\hline Primary or lower & 428 & $(46.4)$ & 149 & $(38.1)$ & 279 & $(52.5)$ \\
\hline Secondary or higher & 494 & $(53.6)$ & 242 & $(61.9)$ & 252 & $(47.5)$ \\
\hline
\end{tabular}


Table 1. Continued

\begin{tabular}{|c|c|c|c|c|c|c|}
\hline \multirow{2}{*}{ CHARACTERISTICS } & \multicolumn{2}{|c|}{ TOTAL ( $\mathrm{N}=931)$} & \multicolumn{2}{|c|}{ MALE $(\mathrm{N}=394)$} & \multicolumn{2}{|c|}{ FEMALE (N = 537) } \\
\hline & $\mathrm{N}$ & $\%$ & $\mathrm{n}$ & $\%$ & $\mathrm{n}$ & $\%$ \\
\hline Speak, write and read Portuguese: & 864 & & 380 & & 484 & \\
\hline Yes & 760 & $(88.0)$ & 352 & $(92.6)$ & 408 & $(84.3)$ \\
\hline No & 104 & $(12.0)$ & 28 & $(7.4)$ & 76 & $(15.7)$ \\
\hline Occupation: & 830 & & 362 & & 468 & \\
\hline Unemployed/ retired & 183 & 22.1 & 54 & 14.9 & 129 & 27.6 \\
\hline Housewife & 51 & 6.1 & 0 & 0 & 51 & 10.9 \\
\hline Full-time Student & 146 & 17.6 & 56 & 15.5 & 90 & 19.2 \\
\hline Employed & 450 & 54.2 & 252 & 69.9 & 198 & 42.3 \\
\hline Household wealth index score, quintiles: & 819 & & 346 & & 473 & \\
\hline Lowest & 109 & 13.3 & 55 & 15.9 & 54 & 11.4 \\
\hline Low & 147 & 18.0 & 61 & 17.6 & 86 & 18.2 \\
\hline Medium & 174 & 21.3 & 76 & 22.0 . & 98 & 20.7 \\
\hline High & 192 & 23.4 & 78 & 22.5 & 114 & 24.1 \\
\hline Highest & 197 & 24.1 & 76 & 22.0 & 121 & 25.6 \\
\hline
\end{tabular}

$\mathrm{IQR}$ - interquartile range

Overall, the median BMI was $22.5(\mathrm{IQR}=20.1,26.2) \mathrm{kg} / \mathrm{m}^{2}$. BMI distribution and behavioral characteristics of study participants are presented in Table 2 with 30.9\% (95\% CI =28.0,33.9) and 12.6\% (95\% CI=10.4, 14.7) of the study participants being overweight + and obese, respectively. Females were characterized by a higher prevalence of overweight and obesity $(42.5 \%$ overweight + and $19.4 \%$ obese) than males $(P<0.0001)$.

Table 2. Behavioral characteristics and BMI distribution of participants $(\mathrm{N}=931)$

\begin{tabular}{|c|c|c|c|c|c|c|c|}
\hline \multirow{2}{*}{ CHARACTERISTICS } & \multicolumn{2}{|c|}{ TOTAL (931) } & \multicolumn{2}{|c|}{ MALE (394) } & \multicolumn{2}{|c|}{ Female (523) } & \multirow[t]{2}{*}{ P-VALUE $\left(\chi^{2}\right)$} \\
\hline & $\mathrm{N}$ & $\%$ & $\mathrm{n}$ & $\%$ & $\mathrm{n}$ & $\%$ & \\
\hline BMI & \multicolumn{2}{|l|}{931} & \multicolumn{2}{|l|}{394} & \multicolumn{2}{|l|}{537} & \multirow{6}{*}{$<0.0001$} \\
\hline Median (IQR): & \multicolumn{2}{|c|}{$22.5(20.1,26.2)$} & \multicolumn{2}{|c|}{$21.2(19.3,23.6)$} & \multicolumn{2}{|c|}{$23.7(20.8,28.3)$} & \\
\hline$<18.5$ & 91 & 9.8 & 57 & 14.4 & 34 & 6.3 & \\
\hline $18.5-24,9$ & 552 & 59.3 & 277 & 70.3 & 275 & 51.2 & \\
\hline $25-29.9$ & 171 & 18.4 & 47 & 13.0 & 124 & 23.1 & \\
\hline$\geq 30$ & 117 & 12.6 & 13 & 3.3 & 104 & 19.4 & \\
\hline Consumption of fruit per week, days: & \multicolumn{2}{|c|}{922} & \multicolumn{2}{|c|}{389} & \multicolumn{2}{|c|}{533} & \\
\hline Median (IQR) & \multicolumn{2}{|c|}{$2(0,3)$} & \multicolumn{2}{|c|}{$2(0,3)$} & \multicolumn{2}{|c|}{$2(0,3)$} & \\
\hline Never & 248 & 26.9 & 107 & 27.5 & 141 & 26.5 & \\
\hline $1-3 \mathrm{~d}$ a week & 488 & 52.9 & 197 & 50.6 & 291 & 54.6 & 0.4284 \\
\hline 4-7 d a week & 186 & 20.2 & 85 & 21.9 & 101 & 19.0 & \\
\hline Consumption of vegetables per week, days: & \multicolumn{2}{|c|}{892} & \multicolumn{2}{|c|}{372} & \multicolumn{2}{|c|}{520} & \\
\hline Median (IQR) & \multicolumn{2}{|c|}{$4(3,6.5)$} & \multicolumn{2}{|c|}{$4(3,6)$} & \multicolumn{2}{|c|}{$4(3,7)$} & \\
\hline Never & 15 & 1.7 & 10 & 2.7 & 5 & 1.0 & \\
\hline 1-3 days a week & 385 & 43.2 & 164 & 44.1 & 221 & 42.5 & 0.1107 \\
\hline 4-7 days a week & 492 & 55.2 & 198 & 53.2 & 294 & 56.5 & \\
\hline Consumption of processed foods per week: & \multicolumn{2}{|c|}{921} & \multicolumn{2}{|c|}{390} & \multicolumn{2}{|c|}{531} & \\
\hline Never & 311 & 33.8 & 132 & 33.9 & 179 & 33.7 & \\
\hline Less than once per week & 369 & 40.1 & 145 & 37.2 & 224 & 42.2 & 0.0867 \\
\hline 1-4 times per week & 175 & 19.0 & 76 & 19.5 & 99 & 18.6 & \\
\hline Daily/ Almost daily & 66 & 7.2 & 37 & 9.5 & 29 & 5.5 & \\
\hline Consumption of soft drinks per week: & \multicolumn{2}{|c|}{894} & \multicolumn{2}{|c|}{373} & & & \\
\hline Never & 507 & 56.7 & 202 & 54.2 & 305 & 58.5 & \\
\hline Less than once per week & 187 & 20.9 & 71 & 19.0 & 116 & 22.3 & 0.0080 \\
\hline 1-4 times per week & 145 & 16.2 & 66 & 17.7 & 79 & 15.2 & \\
\hline Daily/Almost daily & 55 & 6.2 & 34 & 9.1 & 21 & 4.0 & \\
\hline Tobacco consumption & & & & & & & \\
\hline Never consumes & 835 & 90.5 & 319 & 81.8 & 516 & 96.8 & 1 \\
\hline Consumes weekly & 36 & 3.9 & 28 & 7.2 & 8 & 1.5 & $<0.0001$ \\
\hline Consumes daily & 52 & 5.6 & 43 & 11.0 & 9 & 1.7 & \\
\hline
\end{tabular}


Table 2. Continued

\begin{tabular}{|c|c|c|c|c|c|c|c|}
\hline \multirow{2}{*}{ CHARACTERISTICS } & \multicolumn{2}{|c|}{ TOTAL (931) } & \multicolumn{2}{|c|}{ MALE (394) } & \multicolumn{2}{|c|}{ FEMALE (523) } & \multirow[t]{2}{*}{ P-VALue $\left(\chi^{2}\right)$} \\
\hline & $\mathbf{N}$ & $\%$ & $\mathbf{n}$ & $\%$ & $\mathbf{n}$ & $\%$ & \\
\hline Alcohol consumption: & \multicolumn{2}{|c|}{922} & \multicolumn{2}{|c|}{390} & \multicolumn{2}{|c|}{532} & \multirow{5}{*}{$<0.0001$} \\
\hline Never consumed alcohol & 397 & 43.1 & 113 & 29.0 & 284 & 53.4 & \\
\hline Did not consume alcohol last year & 95 & 10.3 & 34 & 8.7 & 61 & 11.5 & \\
\hline Consumes less than once per week & 380 & 41.2 & 205 & 52.6 & 175 & 32.9 & \\
\hline Consumes daily to at least once per week & 50 & 5.4 & 38 & 9.7 & 12 & 2.3 & \\
\hline Number of steps per day & \multicolumn{2}{|c|}{537} & \multicolumn{2}{|c|}{228} & \multicolumn{2}{|c|}{309} & \\
\hline Median (IQR) & \multicolumn{2}{|c|}{$8136(5409,11638)$} & \multicolumn{2}{|c|}{$10166(6668,13866)$} & \multicolumn{2}{|c|}{$6819(4634,9897)$} & \\
\hline$<10000$ & 347 & 64.6 & 110 & 48.3 & 237 & 76.7 & \\
\hline$\geq 10000$ & 190 & 35.4 & 118 & 51.7 & 72 & 23.3 & $<0.0001$ \\
\hline Time spent in MVPA per day, minutes & \multicolumn{2}{|c|}{537} & \multicolumn{2}{|c|}{228} & \multicolumn{2}{|c|}{309} & \\
\hline Median (IQR) & \multicolumn{2}{|c|}{$18,0(6.2,35.0)$} & \multicolumn{2}{|c|}{$24.8(10.2,46.5)$} & \multicolumn{2}{|c|}{$14.0(3.8,28.7)$} & \\
\hline$<60$ & 360 & 67.0 & 128 & 56.1 & 232 & 75.1 & \multirow{2}{*}{$<0.0001$} \\
\hline$\geq 60$ & 177 & 33.0 & 100 & 43.9 & 77 & 24.9 & \\
\hline
\end{tabular}

BMI - body mass index, IQR - interquartile range, MVPA - moderate to vigorous physical activity

The median consumption of fruit and vegetables was on 2 days and 4 days per week, respectively; $26.9 \%$ and $1.7 \%$ of participants never eat fruit and vegetables in a week, respectively. Daily or almost daily consumption of processed foods and soft drinks were reported by $7.2 \%$ and $6.2 \%$ of participants, respectively. There were no statistically significant differences regarding the consumption of fruit and vegetables, processed foods and soft drinks between females and males. Daily tobacco consumption was reported by $5.6 \%$, and $5.4 \%$ of participants reported consuming alcohol daily or at least once per week, with males consuming more tobacco and alcohol than females $(P<0.0001)$. Of those who correctly used pedometers to measure physical activity (ie, throughout the day and over seven days), 64.8\% walked less than the recommended 10000 steps per day, with a median (IQR) of $8136(5409,11638)$ steps per day, while $67.4 \%$ spent less than 60 minutes of MVPA per day, with a median (IQR) of $18(6.2,35)$ minutes of MVPA per day. Females engaged less in physical activity than males $(P<0.0001)$.

Table 3 presents the findings of the univariable modelling. After adjusting for age, females were 2.57 times ( $95 \% \mathrm{CI}=2.02$ to 3.27 ) more likely to be overweight + than males and after adjusting for gender the risk of being overweight + increased 1.03 times $(95 \% \mathrm{CI}=1.03,1.04)$ with each year of age. The highest household wealth index was found to be associated with a 1.92 fold $(95 \% \mathrm{CI}=1.32,2.79)$ increased risk of being over-

Table 3. Associations of overweight+ (BMI $\geq 25)$ with socioeconomic and behavioral characteristics, univariable models and models adjusted for gender and age

\begin{tabular}{|c|c|c|c|c|c|c|c|c|c|}
\hline \multirow{2}{*}{ COVARIATE } & \multirow{2}{*}{$\mathbf{N}$} & \multirow{2}{*}{ EvTs. } & \multirow{2}{*}{ \% EVTS. } & \multicolumn{3}{|c|}{ UNIVARIABLE* } & \multicolumn{3}{|c|}{ ADJ. FOR GENDER \& AGE } \\
\hline & & & & RR & $95 \%$ CI & $P$-value & $\mathrm{RR}$ & $95 \%$ CI & $P$-value \\
\hline \multicolumn{10}{|l|}{ Gender (N=922): } \\
\hline Male & 391 & 59 & 15.09 & 1.00 & - & - & 1.00 & - & - \\
\hline Female & 531 & 223 & 42.00 & 2.78 & $(2.18,3.56)$ & $<0.0001$ & 2.57 & $(2.02,3.27)$ & $<0.0001$ \\
\hline \multicolumn{10}{|l|}{ Age: } \\
\hline Per year & - & - & - & 1.04 & $(1.03,1.04)$ & $<0.0001$ & 1.03 & $(1.03,1.04)$ & $<0.0001$ \\
\hline \multicolumn{10}{|c|}{ Household wealth index score, quintiles $(\mathrm{N}=818)$ : } \\
\hline Very low & 109 & 26 & 23.85 & 1.00 & - & - & 1.00 & - & - \\
\hline Low & 146 & 40 & 27.40 & 1.15 & $(0.72,1.83)$ & 0.5622 & 1.20 & $(0.78,1.86)$ & 0.4127 \\
\hline Medium & 174 & 48 & 27.59 & 1.16 & $(0.75,1.78)$ & 0.5093 & 1.23 & $(0.83,1.83)$ & 0.2997 \\
\hline Medium/high & 192 & 58 & 30.21 & 1.27 & $(0.82,1.96)$ & 0.2906 & 1.35 & $(0.90,2.01)$ & 0.1421 \\
\hline Highest & 197 & 83 & 42.13 & 1.77 & $(1.18,2.65)$ & 0.0062 & 1.92 & $(1.32,2.79)$ & 0.0006 \\
\hline \multicolumn{10}{|l|}{ Education $(\mathrm{N}=921)$ : } \\
\hline Primary or lower & 427 & 171 & 40.05 & 1.00 & - & - & 1.00 & - & - \\
\hline Secondary or higher & 494 & 110 & 22.27 & 0.56 & $(0.45,0.68)$ & $<0.0001$ & 1.03 & $(0.81,1.30)$ & 0.8250 \\
\hline \multicolumn{10}{|c|}{ Marital status $(\mathrm{N}=922)$ : } \\
\hline Single & 471 & 92 & 19.53 & 1.00 & - & - & 1.00 & - & - \\
\hline Married & 392 & 159 & 40.56 & 2.08 & $(1.63,2.65)$ & $<0.0001$ & 1.45 & $(1.13,1.87)$ & 0.0035 \\
\hline Divorced/widow(ed) & 59 & 31 & 52.54 & 2.69 & $(1.96,3.70)$ & $<0.0001$ & 1.18 & $(0.84,1.66)$ & 0.3517 \\
\hline
\end{tabular}


Table 3. Continued

\begin{tabular}{|c|c|c|c|c|c|c|c|c|c|}
\hline \multirow{2}{*}{ COVARIATE } & \multirow{2}{*}{$\mathbf{N}$} & \multirow{2}{*}{ EvTs. } & \multirow{2}{*}{ \% EvTs. } & \multicolumn{3}{|c|}{ UNIVARIABLE* } & \multicolumn{3}{|c|}{ ADJ. FOR GENDER \& AGE } \\
\hline & & & & RR & $95 \%$ CI & $P$-value & RR & $95 \%$ CI & $P$-value \\
\hline \multicolumn{10}{|l|}{ Occupation $(\mathrm{N}=922)$ : } \\
\hline Unemployed/retired & 183 & 50 & 27.32 & 1.00 & - & - & 1.00 & - & - \\
\hline Housewife & 51 & 19 & 37.25 & 1.36 & $(0.87,2.14)$ & 0.1758 & 1.10 & $(0.73,1.68)$ & 0.6445 \\
\hline Student & 146 & 21 & 14.38 & 0.53 & $(0.32,0.85)$ & 0.0095 & 0.94 & $(0.57,1.54)$ & 0.8058 \\
\hline Employed & 449 & 159 & 35.41 & 1.30 & $(0.99,1.70)$ & 0.0623 & 1.51 & $(1.19,1.92)$ & 0.0008 \\
\hline Missing data & 93 & 33 & 35.48 & 1.30 & $(0.89,1.89)$ & 0.1715 & 1.48 & $(1.05,2.07)$ & 0.0236 \\
\hline \multicolumn{10}{|c|}{ Can speak, write and read Portuguese $(\mathrm{N}=922)$ : } \\
\hline No & 103 & 40 & 38.83 & 1.00 & - & - & 1.00 & - & - \\
\hline Yes & 760 & 218 & 28.68 & 0.74 & $(0.56,0.97)$ & 0.0299 & 1.20 & $(0.93,1.56)$ & 0.1627 \\
\hline Missing data & 59 & 24 & 40.68 & 1.05 & $(0.72,1.53)$ & 0.8109 & 0.76 & $(0.53,1.09)$ & 0.1344 \\
\hline \multicolumn{10}{|c|}{ Portuguese as the mother language $(\mathrm{N}=922)$ : } \\
\hline No & 660 & 232 & 35.15 & 1.00 & - & - & 1.00 & - & - \\
\hline Yes & 262 & 50 & 19.08 & 0.54 & $(0.42,0.71)$ & $<0.0001$ & 0.89 & $(0.67,1.19)$ & 0.4469 \\
\hline \multicolumn{10}{|c|}{ Consumption of fruit per week $(\mathrm{N}=919)$ : } \\
\hline Per number of days of the week & - & - & - & 1.04 & $(1.00,1.09)$ & 0.0647 & 1.07 & $(1.03,1.11)$ & 0.0004 \\
\hline \multicolumn{10}{|c|}{ Consumption of fruit per week (N=919): } \\
\hline Never & 247 & 72 & 29.15 & 1.00 & - & - & 1.00 & - & - \\
\hline 1-3 days a week & 486 & 146 & 30.04 & 1.03 & $(0.81,1.31)$ & 0.8041 & 1.18 & $(0.95,1.47)$ & 0.1365 \\
\hline 4-7 days a week & 186 & 64 & 34.41 & 1.18 & $(0.89,1.56)$ & 0.2484 & 1.44 & $(1.11,1.86)$ & 0.0052 \\
\hline \multicolumn{10}{|c|}{ Consumption of vegetables per week $(\mathrm{N}=891)$ : } \\
\hline Per number of days of the week & - & - & - & 1.02 & $(0.98,1.07)$ & 0.3134 & 1.00 & $(0.95,1.04)$ & 0.8330 \\
\hline \multicolumn{10}{|c|}{ Consumption of vegetables per week $(\mathrm{N}=891)$} \\
\hline Never & 15 & 2 & 13.33 & 1.00 & - & - & 1.00 & - & - \\
\hline 1-3 days a week & 385 & 118 & 30.65 & 2.30 & $(0.63,8.33)$ & 0.2050 & 1.91 & $(0.54,6.77)$ & 0.3142 \\
\hline 4-7 days a week & 491 & 160 & 32.59 & 2.44 & $(0.67,8.92)$ & 0.1761 & 1.84 & $(0.51,6.57)$ & 0.3495 \\
\hline \multicolumn{10}{|c|}{ Consumption of processed food per week (N=919): } \\
\hline Never & 310 & 96 & 30.97 & 1.00 & - & & 1.00 & & \\
\hline Less than once per week & 368 & 126 & 34.24 & 1.11 & $(0.89,1.38)$ & 0.3708 & 1.13 & $(0.91,1.39)$ & 0.2686 \\
\hline 1-4 times per week & 175 & 49 & 28.00 & 0.90 & $(0.68,1.21)$ & 0.4969 & 1.15 & $(0.88,1.51)$ & 0.3055 \\
\hline Daily/almost daily & 66 & 10 & 15.15 & 0.49 & $(0.26,0.92)$ & 0.0266 & 0.80 & $(0.44,1.45)$ & 0.4567 \\
\hline \multicolumn{10}{|c|}{ Consumption of soft drinks per week $(\mathrm{N}=893)$ : } \\
\hline Never & 507 & 174 & 34.32 & 1.00 & - & - & 1.00 & - & - \\
\hline Less than once per week & 186 & 58 & 31.18 & 0.91 & $(0.72,1.15)$ & 0.4237 & 1.02 & $(0.82,1.25)$ & 0.8863 \\
\hline 1-4 times per week & 145 & 33 & 22.76 & 0.66 & $(0.48,0.92)$ & 0.0152 & 0.86 & $(0.64,1.16)$ & 0.3345 \\
\hline Daily/almost daily & 55 & 14 & 25.45 & 0.74 & $(0.47,1.17)$ & 0.2005 & 1.08 & $(0.69,1.70)$ & 0.7249 \\
\hline \multicolumn{10}{|l|}{ Tobacco consumption $(\mathrm{N}=920)$ : } \\
\hline Never consumed & 833 & 270 & 32.41 & 1.00 & - & - & 1.00 & - & - \\
\hline Consumes weekly & 36 & 8 & 22.22 & 0.69 & $(0.38,1.24)$ & 0.2113 & 0.95 & $(0.55,1.63)$ & 0.8462 \\
\hline Consumes daily & 51 & 4 & 7.84 & 0.24 & $(0.10,0.57)$ & 0.0013 & 0.27 & $(0.11,0.68)$ & 0.0049 \\
\hline \multicolumn{10}{|l|}{ Alcohol consumption $(\mathrm{N}=919)$ : } \\
\hline Never consumed alcohol & 396 & 121 & 30.56 & 1.00 & - & - & 1.00 & - & - \\
\hline Did not consume alcohol last year & 94 & 37 & 39.36 & 1.29 & $(0.97,1.72)$ & 0.0841 & 1.16 & $(0.91,1.48)$ & 0.2304 \\
\hline Consumes less than once per week & 379 & 116 & 30.61 & 1.00 & $(0.80,1.25)$ & 0.9883 & 1.23 & $(1.00,1.50)$ & 0.0485 \\
\hline $\begin{array}{l}\text { Consumes daily to at least once } \\
\text { per week }\end{array}$ & 50 & 8 & 16.00 & 0.52 & $(0.25,1.09)$ & 0.0825 & 0.90 & $(0.45,1.79)$ & 0.7663 \\
\hline Mean of steps per day $(\mathrm{N}=895)$ : & & & & & & & & & \\
\hline$\leq 10000$ & 347 & 117 & 33.72 & 1.00 & - & - & 1.00 & - & - \\
\hline$>10000$ & 189 & 52 & 27.51 & 0.82 & $(0.62,1.08)$ & 0.1562 & 0.96 & $(0.75,1.24)$ & 0.7779 \\
\hline Missing data & 359 & 111 & 30.92 & 0.92 & $(0.74,1.14)$ & 0.4350 & 0.95 & $(0.78,1.15)$ & 0.5783 \\
\hline Mean of MVPA per day $(\mathrm{N}=895)$ : & & & & & & & & & \\
\hline $60 \mathrm{~min}$ or less & 359 & 117 & 32.59 & 1.00 & - & - & 1.00 & - & - \\
\hline Above $60 \mathrm{~min}$ & 177 & 52 & 29.38 & 0.90 & $(0.70,1.17)$ & 0.4296 & 1.16 & $(0.91,1.49)$ & 0.2245 \\
\hline Missing data & 359 & 111 & 30.92 & 0.95 & $(0.77,1.18)$ & 0.6298 & 1.00 & $(0.82,1.22)$ & 0.9966 \\
\hline
\end{tabular}

$\mathrm{N}$ - number of patients/observations, Evts. - number of overweight and obese patients (BMI>-25), \% Evts.- percentage of overweight and obese patients, RR - risk ratio, $\mathrm{CI}$ - confidence interval

*Associations analyzed using log-link binomial regression with robust variance estimates to adjust for within household clustering. 
weight + compared to the lowest household wealth index category $(P=0.0006)$. Married and employed people were $1.45(95 \% \mathrm{CI}=1.13,1.87)$ and $1.51(95 \%=1.19,1.92)$ times more likely to be overweight + than singles and unemployed participants, respectively. After adjusting for age and gender, the level of education, having Portuguese as one's mother tongue and being able to speak, write and read Portuguese did not show an association with overweight+.

Regarding behavioral risk factors, the consumption of fruit was associated with a 1.07 (95\% CI=1.03, 1.11) times increased risk of overweight + for each additional day $(P=0.0004)$, in contrast to the consumption of processed foods and soft drinks that showed no association with overweight+. Tobacco consumption was found to reduce the risk of being overweight+ $(P=0.0049)$, while alcohol consumption and physical activity were not significantly associated with overweight+.

Appendix S1 in the Online Supplementary Document presents the associations of obesity with socioeconomic and behavioral characteristics. Associations were similar to those described for overweight+, with statistically significant associations between obesity and age, gender and household wealth index and no significant associations for diet, tobacco and physical activity.

Table 4 presents the results for the final multivariable models for overweight+. These show that females were 2.38 times $(95 \% \mathrm{CI}=1.84,3.06)$ more likely to be overweight + than males and that the risk of becoming overweight + increased 1.03 times $(95 \% \mathrm{CI}=1$., 1.03) with every additional year of age. With respect to household wealth, only the highest wealth index category was significantly associated with overweight+, with a 1.98 times (95\% CI $=1.38,2.83$ ) increased risk of overweight + compared to the lowest household wealth index category. Being married and being employed were associated with 1.45 times $(95 \% \mathrm{CI}=1.13,1.86)$ and 1.33 times ( $95 \% \mathrm{CI}=1.04$ to 1.69 ) the risk of overweight+, respectively. Consumption of fruit was associated with a1.05 times $(95 \% \mathrm{CI}=1.01,1.09)$ increased risk of overweight + while daily tobacco consumption was associated with a reduced risk of being overweight+.

Table 4. Multivariable association between overweight + and socioeconomic and behavioral characteristics in Maputo city, Mozambique $(\mathrm{N}=817)$

\begin{tabular}{|c|c|c|c|c|c|c|c|c|c|}
\hline \multirow{2}{*}{ COVARIATE } & \multirow{2}{*}{$\mathbf{N}$} & \multirow{2}{*}{ EvTs. } & \multirow{2}{*}{ \% EvTs. } & \multicolumn{3}{|c|}{ UNIVARIABLE } & \multicolumn{3}{|c|}{ MULTIVARIABLE } \\
\hline & & & & RR & $95 \% \mathrm{CI}$ & $P$-value & RR & $95 \%$ CI & $P$-value \\
\hline \multicolumn{10}{|l|}{ Gender: } \\
\hline Male* & 345 & 55 & 15.94 & 1.00 & - & - & 1.00 & - & - \\
\hline Female & 472 & 200 & 42.37 & 2.66 & $(2.06,3.43)$ & 0.0000 & 2.38 & $(1.84,3.06)$ & $<0.0001$ \\
\hline \multicolumn{10}{|l|}{ Age: } \\
\hline Per year & - & - & - & 1.03 & $(1.03,1.04)$ & 0.0000 & 1.03 & $(1.02,1.03)$ & $<0.0001$ \\
\hline \multicolumn{10}{|l|}{ Household wealth index, quintiles: } \\
\hline Very low* & 109 & 26 & 23.85 & 1.00 & - & - & 1.00 & - & - \\
\hline Low & 146 & 40 & 27.40 & 1.15 & $(0.72,1.83)$ & 0.5622 & 1.29 & $(0.86,1.95)$ & 0.2161 \\
\hline Medium & 174 & 48 & 27.59 & 1.16 & $(0.75,1.78)$ & 0.5093 & 1.30 & $(0.89,1.89)$ & 0.1758 \\
\hline Medium/high & 192 & 58 & 30.21 & 1.27 & $(0.82,1.96)$ & 0.2906 & 1.35 & $(0.92,1.98)$ & 0.1206 \\
\hline Highest & 196 & 83 & 42.35 & 1.78 & $(1.18,2.67)$ & 0.0058 & 1.98 & $(1.38,2.83)$ & 0.0002 \\
\hline \multicolumn{10}{|l|}{ Marital status: } \\
\hline Single* & 402 & 81 & 20.15 & 1.00 & - & - & 1.00 & - & - \\
\hline Married & 357 & 143 & 40.06 & 1.99 & $(1.54,2.56)$ & 0.0000 & 1.45 & $(1.13,1.86)$ & 0.0033 \\
\hline Divorced/widow(ed) & 58 & 31 & 53.45 & 2.65 & $(1.92,3.66)$ & 0.0000 & 1.21 & $(0.87,1.68)$ & 0.2557 \\
\hline \multicolumn{10}{|l|}{ Occupation: } \\
\hline Retired/unemployed* & 160 & 47 & 29.37 & 1.00 & - & - & 1.00 & - & - \\
\hline Housewife & 45 & 16 & 35.56 & 1.21 & $(0.74,1.97)$ & 0.4412 & 0.96 & $(0.61,1.52)$ & 0.8737 \\
\hline Student & 132 & 19 & 14.39 & 0.49 & $(0.30,0.81)$ & 0.0054 & 0.87 & $(0.52,1.45)$ & 0.5956 \\
\hline Employed & 402 & 142 & 35.32 & 1.20 & $(0.91,1.60)$ & 0.2020 & 1.33 & $(1.04,1.69)$ & 0.0217 \\
\hline Missing data & 78 & 31 & 39.74 & 1.35 & $(0.93,1.96)$ & 0.1108 & 1.54 & $(1.12,2.12)$ & 0.0084 \\
\hline \multicolumn{10}{|l|}{ Consumption of fruit per week: } \\
\hline Per number of days of the week & - & - & - & 1.04 & $(1.00,1.09)$ & 0.0602 & 1.05 & $(1.01,1.09)$ & 0.0175 \\
\hline \multicolumn{10}{|l|}{ Tobacco consumption: } \\
\hline Never consumed* & 743 & 244 & 32.84 & 1.00 & - & - & 1.00 & - & - \\
\hline Consumes weekly & 31 & 8 & 25.81 & 0.79 & $(0.44,1.40)$ & 0.4127 & 0.89 & $(0.52,1.51)$ & 0.6584 \\
\hline Consumes daily & 43 & 3 & 6.98 & 0.21 & $(0.08,0.59)$ & 0.0032 & 0.25 & $(0.09,0.72)$ & 0.0102 \\
\hline
\end{tabular}

$\mathrm{N}$ - number of observations, Evts. - number of events, \% Evts. - (N-events/N) $\times 100$, RR - risk ratio, CI - confidence interval

* Reference stratum. 
Appendix S2 in the Online Supplementary Document presents the results for the final multivariable models for obesity where, similar to overweight, being female, increasing age and a greater household wealth index were associated with a higher risk of obesity. Higher levels of education were associated with a significantly reduced risk of being obese.

\section{DISCUSSION}

\section{Key findings}

Previous studies carried out in Mozambique have described an increase of overweight and obesity prevalence, especially in urban areas $[6-8,10]$. The purpose of this study was to determine the prevalence of overweight and obesity and their association with social and behavioral determinants in the capital city of Mozambique, where urbanization is progressing rapidly and disorderly, with a visible reduction of recreation spaces and no active promotion of healthy lifestyle habits. We found that among youth and adults from the peri-urban area of Maputo city, one in every three is overweight and more than one in every ten is obese. Females, the elderly and people with greater household wealth have a significantly increased risk of being overweight and obese compared to males, younger people and individuals with lower household wealth index. As documented in previous studies [23-25], tobacco consumption is associated with a reduced risk of being overweight, and those with higher levels of education have a reduced risk of obesity compared to those with lower levels of education. Put simply, this suggests that - in this specific context of a peri-urban area of Maputo - being wealthier increases the risk of certain unhealthy lifestyle habits that enhance the risk of gaining weight, whereas being more educated appears to counteract the risk of gaining weight.

\section{Locating findings in the literature}

The high levels of overweight and obesity found in this study are in accordance with data from Mozambique [6-8] and other SSA countries [26-31], and show once again that even countries with low levels of national wealth and urbanization such as Mozambique are facing the burden of overweight and obesity [32].

Similar to findings from the Mozambican STEPS surveys [7,8] and from other African countries [27,28,31,3335], being female and being older are strong predictors of overweight and obesity, with risks of overweight and obesity being 2 times and more than 5 times greater among women compared to men, respectively. This difference between sexes has been previously explained by gender differences in metabolism and hormonal balance [36], among other cultural and behavioral reasons.

In agreement with previous studies that showed an association between household wealth and overweight in Africa, including in Mozambique [7,8,27,28,32,33,37,38], our study shows a strong association of household wealth with overweight and obesity, where participants in households with the highest household wealth index have an almost two times and a five times elevated risk of being overweight or obese respectively, compared to those from households with the lowest household wealth index.

Our results showed that married individuals are more likely to be overweight than singles - a finding that does not differ from previous studies from SSA $[30,39]$.

In line with a previous study conducted in other SSA countries [30], our findings showed that higher levels of education seem to be protective against obesity, which might be related to the fact that more educated people are more informed regarding NCDs and associated risk factors, as well as health-protective behaviors. In contrast, those that are employed are more likely to be overweight compared to unemployed youth and adults, which might be explained by the fact that employed people from this study were more likely to consume processed and often high-energy foods.

Our findings did not show a decreased risk of overweight and obesity in those with a higher consumption of fruit and vegetables [40] as we would have expected. Conversely, it showed an increased risk of being overweight with an increase in the number of days on which fruit and vegetables are consumed.

Even in less urbanized countries such as Mozambique, urban residents purchase most of their food, whereas rural residents base their diet primarily on family farming. City dwellers from Maputo buy $92 \%$ of their food, which increases the probability of consuming pre-processed high energy foods [41]. Soft drinks and fast food are usually related to obesity due to their high energy content [42], however, this study did not find any association between BMI and consumption of processed food and consumption of soft drinks. Thus, the study 
documents the frequency of consumption of "junk food", which can be used as a baseline for monitoring the dynamics of change in diet and can also provide evidence to the development of policies for the reduction of saturated fatty acids and trans-fats in Mozambique [5].

Physical activity is commonly described as a protective factor for overweight and obesity [43], including in SSA [27]. Although the study population showed low levels of physical activity (particularly among women), we did not find any association between physical activity and BMI. The relationship between BMI and physical activity is influenced by a complex combination of factors such as time spent watching television, environmental conditions to practice physical activity, urban crime and other predictors of physical inactivity [44], which are likely to play a role in explaining this result.

Studies from other African countries addressed the perception of ideal body image, where overweight individuals were found to be perceived as healthier, wealthier or prettier $[35,39,45,46]$, making the problem of overweight and obesity even more complex in such environments. Understanding the social and behavioral function and predictors of overweight and obesity in the local context is crucial for designing and implementing interventions to reduce the prevalence of overweight and obesity in Mozambique and in other developing countries and, consequently, to prevent NCDs. Meanwhile, studies regarding cultural factors, beliefs and perceptions about body weight are needed in order to develop culturally appropriate new policies and to adapt existing ones to the local context in order to achieve the goals stated in the Global Action Plan for NCDs and the Sustainable Development Goals.

\section{Strengths and limitations}

One of the major limitations of this study is its cross-sectional design that does not allow us to infer causality. Although the exclusion of 32 participants (3.3\% of those who were eligible and provided informed consent) without anthropometric measurements could have been an important shortcoming, it is unlikely to have had a big influence on our effect estimates. As previous data described multiparous women to be at a higher risk of increased BMI [47], the fact that our study was unable to collect information regarding parity represents another limitation. We also recognize limitations related to the diet component of our study: first, the consumption of fruit and vegetables was not measured per unit, but per number of days consuming fruit and vegetables, due to the difficulty of the interviewees to recall this in detail; second, as this was not a dietary survey and hence lacks information regarding the consumption of saturated fat, animal protein and sugar, important elements of the diet of the population under study [48].

Nevertheless, this study reports substantial and reliable data regarding BMI from participants in a peri-urban developing country setting. With rapid urbanization Such peri-urban populations will represent the majority of the population in countries like Mozambique in the near future. Although this paper was focused on overweight and obesity, it also shows that underweight should not be neglected in adults ( 1 in every 10 adults were underweight), especially due to the high burden of HIV and tuberculosis [49]. Other strengths of this study are related to its methodology: a large sample size was achieved, allowing comparison of groups; context-sensitive wealth index quintiles were obtained through robust analysis; validated data collection tools from the STEPS survey were adapted to the local context; and objective measurements (using pedometers and accelerometers) were used to describe physical activity.

\section{CONCLUSION}

In summary, the high prevalence of overweight and obesity and the low levels of physical activity and low intake of fruits and vegetables in peri-urban areas of Maputo city are of considerable public health concern. The statistically significant relationship between overweight/obesity and female sex, older age and higher household wealth highlights the need for focused and culturally appropriate interventions in this population to reduce the burden of overweight and obesity in Maputo city, Mozambique. NCD prevention and control measures focused on individual lifestyle and environmental factors to promote a healthier diet and increased physical activity are urgently needed. 


Acknowledgments: The authors acknowledge the Polana Caniço community, especially the study participants and the
staff at the Centro de Investigação e Treino em Saúde da Polana Caniço (CISPOC) and at the Centro de Investigação do Desporto
e da Actividade Física (CIDAF) for their collaboration in this project, with special thanks offered to Jorge Uate, Paulino da
Costa, Regina Timana, Vânia Maphossa, and the HDSS Polana Caniço team.
Funding: This research was funded, in part, by the Flemish Government through BICMINS project: Building Institu-
tional Capacity at the Mozambique INS. There is no objection to the publication and/or presentation of this research. The
funders had no role in study design, data collection and analysis, or preparation of the manuscript.
Authorship contributions: IM was primarily responsible for conceptualizing the study with support from AP, KP and
ER. IM and AP were responsible for study implementation with support from NC, CM and SN. IM and ES led data anal-
ysis with support from NC and ER. IM prepared the first draft of the manuscript with support from AP, KP, ER and ES.
All authors reviewed and approved the final version of the manuscript.
Competing interests: The authors completed the ICMJE Unified Competing Interest form (available upon request from
the corresponding author), and declare no conflicts of interest.
Additional material
Online Supplementary Document

1 WHO Global status report on noncommunicable diseases. Geneva: WHO; 2014.

2 Manyanga T, El-sayed H, Doku DT, Randall JR. The prevalence of underweight, overweight, obesity and associated risk factors among school-going adolescents in seven African countries. BMC Public Health. 2014;14:887. Medline:25168589 doi:10.1186/1471-2458-14-887

3 World Health Organization. Global action plan for the prevention and control of noncommunicable diseases 2013-2020. Geneva: WHO; 2013.

4 WHO. Status of the health-related SDGs. 2018.

5 Riley L, Gouda H, Cowan M. Noncomminicable Diseases Progress Monitor 2017. Geneva: WHO; 2017.

6 dos Santos FK, Maia JAR, Gomes TNQF, Daca T, Madeira A, Katzmarzyk PT, et al. Secular trends in growth and nutritional status of Mozambican school-aged children and adolescents. PLoS One. 2014;9:e114068. Medline:25473837 doi:10.1371/ journal.pone.0114068

7 Fontes F, Damasceno A, Jessen N, Silva-matos C, Padrão P, Lunet N. Prevalence of overweight and obesity in Mozambique in 2005 and 2015. Public Health Nutr. 2019;22:3118-26. Medline:31453793 doi:10.1017/S1368980019002325

8 Gomes A, Damasceno A, Azevedo A, Prista A, Silva-Matos C, Saranga S, et al. Body mass index and waist circumference in Mozambique: Urban/rural gap during epidemiological transition. Obes Rev. 2010;11:627-34. Medline:20406415 doi:10.1111/ j.1467-789X.2010.00739.x

9 Nhantumbo L, Manasse S. Alterações no padrão de actividade física em função da urbanização e determinantes socio-culturais: um estudo em crianças e jovens de maputo (moçambique). Rev Bras Ciência e Mov. 2009;16:25-32.

10 Prista A. Sedentarismo, urbanização e transição epidemiológica. Rev Cient da UEM. 2012;1:28-38.

11 Maputo CM de. Perfil Estatístico Do Município de Maputo. 2010.

12 Vyas S, Kumaranayake L. Constructing socio-economic status indices: How to use principal components analysis. Health Policy Plan. 2006;21:459-68. Medline:17030551 doi:10.1093/heapol/czl029

13 Riley L, Guthold R, Cowan M, Savin S, Bhatti L, Armstrong T, et al. The World Health Organization STEPwise Approach to Noncommunicable Disease Risk-Factor Surveillance: Methods, Challenges, and Opportunities. Am J Public Health. 2016;106:748. Medline:26696288 doi:10.2105/AJPH.2015.302962

14 Carlson RV, Boyd KM, Webb DJ. The revision of the Declaration of Helsinki: Past, present and future. Br J Clin Pharmacol. 2004;57:695-713. Medline:15151515 doi:10.1111/j.1365-2125.2004.02103.x

15 Cuschieri S. The STROBE guidelines. Saudi J Anesth. 2019;31-4.

16 Lim SS, Vos T, Flaxman AD, Danaei G, Shibuya K, Adair-Rohani H, et al. A comparative risk assessment of burden of disease and injury attributable to 67 risk factors and risk factor clusters in 21 regions, 1990-2010: a systematic analysis for the Global Burden of Disease Study 2010. Lancet. 2012;380:2224-60. Medline:23245609 doi:10.1016/S0140-6736(12)61766-8

17 Filmer D, Pritchett LH. Estimating wealth effects without expenditure data - Or tears: An application to educational enrollments in states of India. Demography. 2001;38:115-32. Medline:11227840

18 Kolenikov AG. Socioeconomic status measurement with discrete proxy variables: is principal component analysis a reliable answer? Rev Income Wealth. 2009;55: 128-65. doi:10.1111/j.1475-4991.2008.00309.x

19 Nuttall FQ. Body Mass Index: Obesity, BMI, and Health - a critical review. Nutr Today. 2015;50:117-28. Medline:27340299 doi:10.1097/NT.0000000000000092

20 Barros AJD, Hirakata VN. Alternatives for logistic regression in cross-sectional studies: An empirical comparison of models that directly estimate the prevalence ratio. BMC Med Res Methodol. 2003;3:21. Medline:14567763 doi:10.1186/1471-2288-3-21

21 McNutt LA, Wu C, Xue X, Hafner JP. Estimating the relative risk in cohort studies and clinical trials of common outcomes. Am J Epidemiol. 2003;157:940-3. Medline:12746247 doi:10.1093/aje/kwg074

22 Stata Press. STATA Base Reference Manual Release 13. 2013. 
23 Jacobs M. Addictive Behaviors Reports Adolescent smoking: The relationship between cigarette consumption and BMI. Addict Behav Rep. 2018;9:100153. Medline:31193813

24 Chiolero A, Faeh D, Paccaud FCJ. Consequences of smoking for body weight, body fat distribution. Am J Clin Nutr. 2008;87:8019. Medline:18400700 doi:10.1093/ajcn/87.4.801

25 Dare S, Mackay DF, Pell JP. Relationship between Smoking and Obesity: A Cross-Sectional Study of 499, 504 Middle- Aged Adults in the UK General Population. PLoS One. 2015;10:e0123579. Medline:25886648 doi:10.1371/journal.pone.0123579

26 Peer N, Lombard C, Steyn K, Gwebushe N, Levitt N. Differing patterns of overweight and obesity among black men and women in Cape Town: The CRIBSA study. PLoS One. 2014;9:e107471. Medline:25221948 doi:10.1371/journal.pone.0107471

27 Steyn NP, Mchiza ZJ. Obesity and the nutrition transition in Sub-Saharan Africa. Ann N Y Acad Sci. 2014;1311:88-101. Medline:24725148 doi:10.1111/nyas. 12433

28 Ajayi IO, Adebamowo C, Adami H, Dalal S, Diamond MB, Bajunirwe F, et al. Urban - rural and geographic differences in overweight and obesity in four sub-Saharan African adult populations: a multi-country cross-sectional study. BMC Public Health. 2016;16:1126. Medline:27793143 doi:10.1186/s12889-016-3789-z

29 Neupane S, Prakash KC, Doku DT. Overweight and obesity among women: analysis of demographic and health survey data from 32 Sub-Saharan African Countries. BMC Public Health. 2016;16:30. Medline:26758204 doi:10.1186/s12889-016-2698-5

30 Ziraba AK, Fotso JC, Ochako R. Overweight and obesity in urban Africa: A problem of the rich or the poor? BMC Public Health. 2009;9:465. Medline:20003478 doi:10.1186/1471-2458-9-465

31 Baalwa J, Bb B, Ke K, Am O. Prevalence of overweight and obesity in young adults in Uganda. Afr Health Sci. 2010;10:36773. Medline:21416039

32 Madise NJ, Letamo G. Complex association between rural/urban residence, household wealth and women's overweight: Evidence from 30 crosssectional national household surveys in africa. BMC Obes. 2017:4:5. Medline:28127440 doi:10.1186/ s40608-016-0141-1

33 Kirunda BE, Fadnes LT, Wamani H, Van Den Broeck J, Tylleskär T. Population-based survey of overweight and obesity and the associated factors in peri-urban and rural Eastern Uganda. BMC Public Health. 2015;15:1168. Medline:26602893 doi:10.1186/ s12889-015-2506-7

34 Ofori-Asenso R, Agyeman AA, Laar A, Boateng D. Overweight and obesity epidemic in Ghana — a systematic review and meta-analysis. BMC Public Health. 2016;16:1239. Medline:27938360 doi:10.1186/s12889-016-3901-4

35 Ettarh R, Van De Vijver S, Oti S. Overweight, Obesity, and Perception of Body Image Among Slum Residents in Nairobi, Kenya, 2008 - 2009. Prev Chronic Dis. 2013;10:E212. Medline:24355105 doi:10.5888/pcd10.130198

36 Lovejoy JC, Sainsbury A, Working C. Sex differences in obesity and the regulation of energy homeostasis. Obes Rev. 2009;10:15467. Medline:19021872 doi:10.1111/j.1467-789X.2008.00529.x

37 Prista A, Maia JAR, Damasceno A, Beunen G. Anthropometric indicators of nutritional status: implications for fitness, activity, and health in school-age children and adolescents from Maputo, Mozambique. Am J Clin Nutr. 2003;77:952-9. Medline:12663297 doi:10.1093/ajcn/77.4.952

38 Prista A, Maia AJR, Saranga S, Nhantumbo L, Marques AT, Beunen G. Somatic growth of a school-aged population from Mozambique: Trend and biosocial meaning. Hum Biol. 2005;77:457-70. Medline:16485776 doi:10.1353/hub.2005.0069

39 Benkeser RM, Biritwum R, Hill AG. Prevalence of overweight and obesity and perceptions of healthy and desirable body size in urban, Ghanaian women. Ghana Med J. 2012;46:66-75. Medline:22942454

40 Nishida C, Uauy R, Kumanyika S, Shetty P. The Joint WHO / FAO Expert Consultation on diet, nutrition and the prevention of chronic diseases: process, product and policy implications. Public Health Nutr. 2004;7:245-50. Medline:14972063 doi:10.1079/PHN2003592

41 Nishida C, Uauy R, Kumanyika S, Shetty P; Food and Agriculture Organization of the United Nations. Globalization of food systems in developing countries: impact on food, security and nutrition. 2004;83:1-300

42 Denney-Wilson E, Crawford D, Dobbins T, Hardy L, Ded ADO. Influences on consumption of soft drinks and fast foods in adolescents. Asia Pac J Clin Nutr. 2009;18:447-52. Medline:19786394

43 Chin SH, Kahathuduwa CN, Binks M. Physical activity and obesity: what we know and what we need to know? Obes Rev. 2016;17:1226-44. Medline:27743411 doi:10.1111/obr.12460

44 Kruger HS, Puoane T, Senekal M, Van Der Merwe M. Obesity in South Africa: challenges for government and health professionals. Public Health Nutr. 2005;8:491-500. Medline:16153330 doi:10.1079/PHN2005785

45 Wittenberg M. The Weight of Success: The Body Mass Index and Economic Well-being in South Africa. 2005.Available: http:// ipc.umich.edu/sites/all/themes/ipc_umfs_subtheme/images/edts/pdfs/wittenberg.pdf. Accessed: 10 December 2018.

46 In EW, Africa S. The weight of sucess: the body mass index and economic well-being in Southern Africa. Rev Income Wealth. 2015;59 Suppl 1:1-28.

47 Heliövaara M, Aromaa A. Parity and obesity. J Epidemiol Community Health. 1981;35:197-9. Medline:7328379 doi:10.1136/ jech.35.3.197

48 Popkin BM, Corvalan C, Grummer-strawn LM. Series Double Burden of Malnutrition 1 Dynamics of the double burden of malnutrition and the changing nutrition reality. Lancet. 2020;395:65-74. Medline:31852602 doi:10.1016/S0140-6736(19)32497-3

49 USAID. Mozambique: Nutrition Profile. 2018. Available: https://www.usaid.gov/sites/default/files/documents/1864/Mozambique-Nutrition-Profile-Mar2018-508.pdf. Accessed: 10 December 2018. 